\title{
Using X-band Weather Radar Measurements to Monitor the Integrity of Digital Elevation Models for Synthetic Vision Systems
}

\author{
Steve Young ${ }^{\mathrm{a}}$, Maarten Uijt de Haag ${ }^{\mathrm{b}}$, Jonathon Sayre ${ }^{\mathrm{b}}$ \\ ${ }^{a}$ NASA Langley Research Center, ${ }^{b}$ Ohio University
}

\begin{abstract}
Synthetic Vision Systems (SVS) provide pilots with displays of stored geo-spatial data representing terrain, obstacles, and cultural features. As comprehensive validation is impractical, these databases typically have no quantifiable level of integrity. Further, updates to the databases may not be provided as changes occur. These issues limit the certification level and constrain the operational context of SVS for civil aviation. Previous work demonstrated the feasibility of using a realtime monitor to bound the integrity of Digital Elevation Models (DEMs) by using radar altimeter measurements during flight. This paper describes an extension of this concept to include X-band Weather Radar (WxR) measurements. This enables the monitor to detect additional classes of DEM errors and to reduce the exposure time associated with integrity threats. Feature extraction techniques are used along with a statistical assessment of similarity measures between the sensed and stored features that are detected. Recent flight-testing in the area around the Juneau, Alaska Airport (JNU) has resulted in a comprehensive set of sensor data that is being used to assess the feasibility of the proposed monitor technology. Initial results of this assessment are presented.
\end{abstract}

Keywords: synthetic vision systems, digital elevation models, radar, integrity, feature detection.

\section{INTRODUCTION}

SVS provides pilots with a virtual visual depiction of the external environment. This depiction can be portrayed on any flight-qualified display media. Prototype implementations have used both head-down displays and head-up displays to provide pilots with aircraft state (e.g. altitude, attitude, airspeed), guidance and navigation information, and a perspective depiction of the terrain as viewed from the cockpit. Other types of information can also be presented such as obstacles, traffic, and weather. SVS has the potential to improve flight safety by increasing pilot situational awareness (SA) in low visibility conditions to a level of awareness similar to daytime clear-weather flying. NASA has been investigating SVS as a mitigation strategy for accident categories such as Controlled Flight Into Terrain (CFIT), runway incursions, low visibility and loss-of-control scenarios; along with the ability to fly advanced precision approach procedures. In addition, the Federal Aviation Administration (FAA) has mandated Terrain Awareness and Warning Systems (TAWS) for nearly all aircraft to increase pilot's SA with respect to terrain. The major difference between TAWS and SVS is that SVS is being designed for applications ranging from purely advisory systems to flight-critical systems, whereas TAWS is currently required only as an advisory system.

\section{$1.1 \quad$ Integrity}

The term integrity is used frequently in the aviation community as a quality metric. Several segments of the community interpret integrity differently. There are three definitions of integrity that are relevant to the research presented in this paper: system integrity, data integrity, and data processing integrity. With respect to DEMs used by SVS, required data integrity will depend on the intended use of the data by the pilot and the architecture of the system in which the data resides. DEM integrity is related to system integrity in that system integrity can be compromised if errors exist in the DEM that may lead to Hazardous Misleading Information (HMI) being presented to pilots, and these errors are not detected by the operational system.

To ensure that data is not corrupted during processing and/or distribution, the International Civil Aviation Organization (ICAO) has established guidelines for data processing integrity. ICAO defines data processing integrity as the degree of assurance that aeronautical data and its value have not been altered since the data origination or an authorized amendment [1]. To provide guidance to data processors and/or distributors, RTCA has published guidelines for data processing procedures that are intended to help ensure that the resulting data is no worse than the source data [2]. It is expected that the 
majority of terrain data that is stored on aircraft as part of an SVS or TAWS will not have a stated integrity with respect to the source data itself. The integrity specified with these data will only refer to data processing integrity. This is primarily due to the fact that the amount of validation required to establish an integrity value for such large data sets is viewed as cost prohibitive.

\subsection{Digital elevation models}

Geo-spatial terrain databases, that may be used to depict terrain information on SVS displays, are referred to as Digital Elevation Models (DEMs). A variety of sources from both the public and private sectors provide DEMs, and these DEMs are specified by a number of parameters. These parameters include the post-spacing or spatial resolution of elevation measurements, the horizontal and vertical references or datums, and the circular and linear error probabilities. The circular error probability (CEP) is used for the horizontal accuracy specification of the post location, whereas the linear error probability (LEP) is used to specify the accuracy in the vertical direction for the post elevation. Assuming that the circular and linear errors are random and normally distributed with zero-mean, the standard deviations in the horizontal and vertical directions, can be derived by dividing the $90 \%$ CEP and 90\% LEP by 1.645 . The standard deviation in the horizontal direction refers to the standard deviation of the error radius from the true post location using a two-dimensional polar coordinate frame.

\subsection{Integrity monitoring approach}

When utilizing DEMs in flight-critical systems, it is imperative to avoid the display of hazardous misleading terrain information (HMTI). HMTI can be the result of insufficient DEM spatial resolution, inappropriate coloring/texturing, or excessive DEM errors. The severity of the hazard will depend on the specific flight operation being conducted and the use of the terrain depiction by pilots during this operation. This paper describes an approach to real-time terrain database integrity monitoring that can reduce the probability of an undetected database error being inadvertently presented to the pilot. The proposed integrity monitor concept is an extension to the approach described in [3][4][5]. For the proposed monitor, sensor information from the Global Positioning System (GPS), an Inertial Reference Unit (IRU), and an X-band WxR are used in connection with a DEM to enable feature-based disparity checking. Disparities between features that are sensed and features that are extracted from the stored DEM are compared in a statistical manner similar to the one described in [5]. If significant inconsistencies exist, an integrity alert is generated. The remainder of this paper provides specifics on how this can be achieved by making use of a large data set that was recently acquired during flight-testing in the JNU region.

\section{X-BAND WEATHER RADAR OVERVIEW}

Previous work has shown how radar altimeters can be integrated with SVS to provide a level of integrity for a DEM [3][4][5]. Unfortunately, the radar altimeter approach has two shortcomings. First, because radar altimeters make measurements nominally from nadir, looking-down vertically, horizontal DEM errors are difficult to detect unless there is significant terrain undulation under the flight path. Second, unless the detected error is a bias or ramp-type error that persists over a large area of the DEM, the detection may not be operationally useful as the aircraft has already flown over the region where the error was detected. Both of these shortcomings led to the need to look at forward-looking sensors.

In today's aircraft fleet, by far the most common forward-looking sensor is the weather radar. Specifically, X-band pulse Doppler radars are used primarily to provide flight crews with a display of weather information and to provide forwardlooking wind-shear detection capability. A secondary purpose, as described in [6], is "ground-mapping to facilitate navigation by display of significant land contours". Although using the weather radar display to aid navigation was historically a pilot-specific talent derived from extensive use of the device, recent work has shown that integration with DEMs can be used to supplement on-board navigation systems and to detect potential ground-based hazards [7][8][9].

A typical certified WxR system includes a Receiver/Transmitter $(\mathrm{R} / \mathrm{T})$, an antenna, a radome, and a display. The WxR system is designed such that all signal processing is performed by the $\mathrm{R} / \mathrm{T}$ and data products are provided to the display. As examples, the radome, antenna, and R/T for NASA's B-757 are shown in Figure 1. The R/T installed on this aircraft is a Rockwell-Collins WxR-2100.

Details on the characteristics of commercial certified WxR systems can be found in [6][10][11]. Operating frequencies for X-band systems range from 9.36 to $9.56 \mathrm{GHz}$. Antenna diameters vary from 15 to 30 inches with gains ranging from 28.9 to 
$35 \mathrm{~dB}$, respectively. Beam-width can vary from 2.9 to 6.0 degrees. Antennas scan up to $+/-90$ degrees with respect to the longitudinal axis of the aircraft at a nominal rate of 15 looks per minute ( 45 degrees per second). Range can be set by the pilot to $5,10,20,40,80,160$, or $320 \mathrm{nmi}$; however, the number of range bins with reported reflectivity measurements remains fixed at 512. Each reflectivity measurement is quantized into four levels with a fifth used for severe windshear. For display, colors are associated with these levels for each range bin during scanning. Measurement interval time is nominally 6 msec using a 0.25 degree azimuth resolution during scanning. The above specifications are taken from [6].
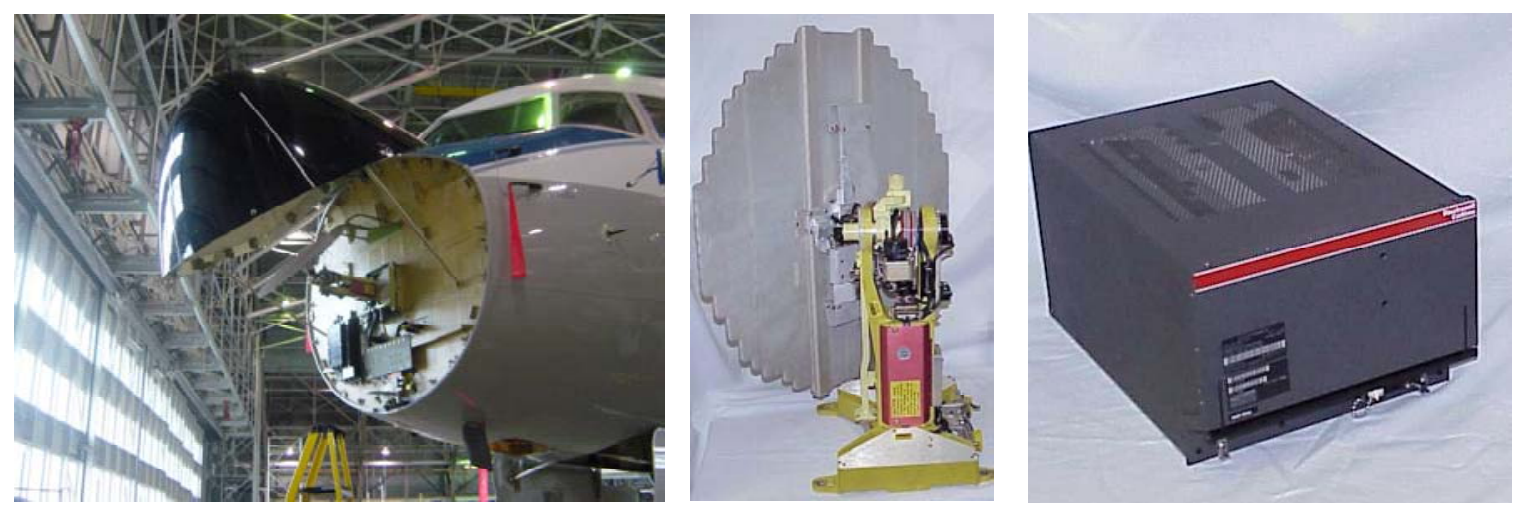

Figure 1. NASA B-757 radome, antenna, and WxR-2100 R/T

\section{FEATURE-BASED DISPARITY CHECKING}

Because the WxR provides measurements at a high rate, it is desirable to extract terrain-specific features from these measurements to reduce the complexity of the disparity checking (i.e. integrity monitoring) function. In addition, to quantify integrity and/or integrity bounds, a statistical approach is required. Because solving statistical problems involving combinations of several random variables can be intractable, a reduced, or minimum, set of features to use for disparity checking is desired.

Figure 2 depicts the proposed algorithm for feature-based disparity checking using a forward-looking scanning sensor such as the WxR. Using this approach, there are two threads that operate in parallel: a sensor-derived thread and a DEM-derived thread. Ideally these threads would be completely independent to eliminate the possibility of a common-mode failure that may not be detectable. In Figure 2, the sensor-derived thread provides the sensor pointing direction (AZANG, TILT) and the range setting to the DEM-derived thread. This inter-dependence, while not ideal, should not compromise integrity since failures of these data elements should be detectable (e.g. AZANG not changing during scanning).

The first two stages of each thread are responsible for detecting features along each radial line measurement (i.e. within the 512 range bins) then classifying these features. For the prototype version of the algorithm, shadow edge locations are used as the features of interest. Shadow edges are often the most significant feature discernable in WxR measurements and occur when reflectivity values transition to/from $0 \mathrm{~dB}$ indicating no detectable reflectivity. WxR shadowing occurs most frequently in areas of moderate to severe terrain undulations when the aircraft is at a relatively low altitude or the antenna depression angle (i.e. tilt) is large. Figure 3 depicts a segment of a sample WxR radial measurement and the edge features that would be detected and classified as either rising or falling edges by the prototype algorithm. The gradient at each edge can also be computed and used as a weighting factor to down-sample features prior to disparity checking.

Feature detection and classification in the DEM-derived thread is similar; however, the challenge for this thread is to generate synthesized radial measurements. The prototype algorithm uses aircraft position from GPS, aircraft attitude from an IRU, antenna pointing direction from the WxR, a beam model, and a DEM to generate the synthesized measurements. Each measurement is created by finding the DEM height at each range bin location (1-512) along the azimuth pointing direction of the antenna, then finding the depression angle from these points on the DEM back to the WxR antenna location. These depression angles are then compared to the expected range of angles for the main beam for a particular measurement. The expected range of angles is tilt $+/$ - one-half of the beam-width. Each range bin for a synthesized measurement is assigned a 
value as follows: -3 , denotes the DEM-derived depression angle is outside the range of the main beam; 1 , denotes the angle is within the range of the main beam and therefore, energy should be reflected back to the WxR; 2, denotes an edge location where the angle has passed through a local minima; and 0, denotes a shadowed range bin. Using this synthesized measurement, edge features can be detected and classified by the identical method used for the actual WxR measurements in the WxR-derived thread.

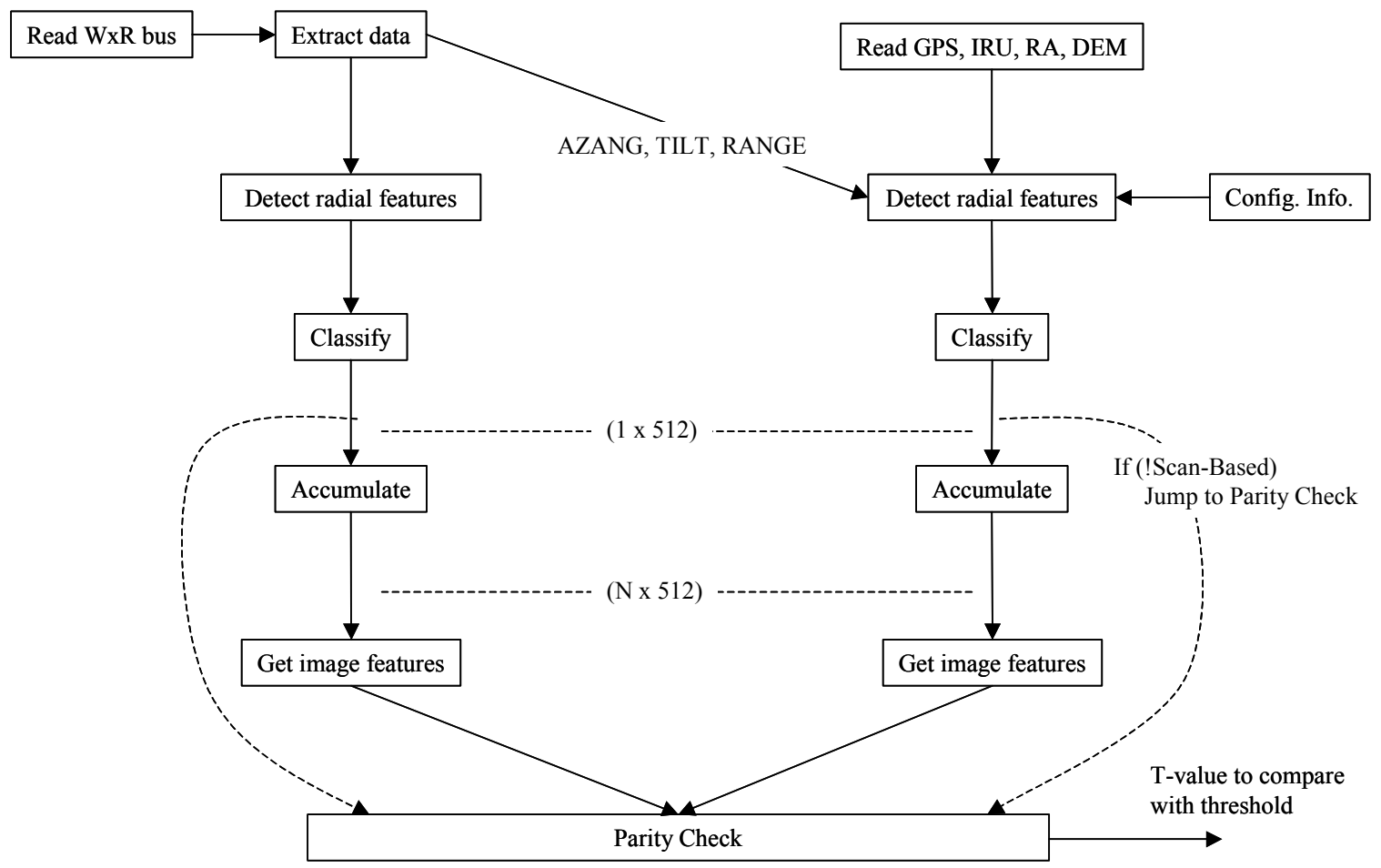

Figure 2. Feature-based disparity checking algorithm using forward-looking scanning sensor

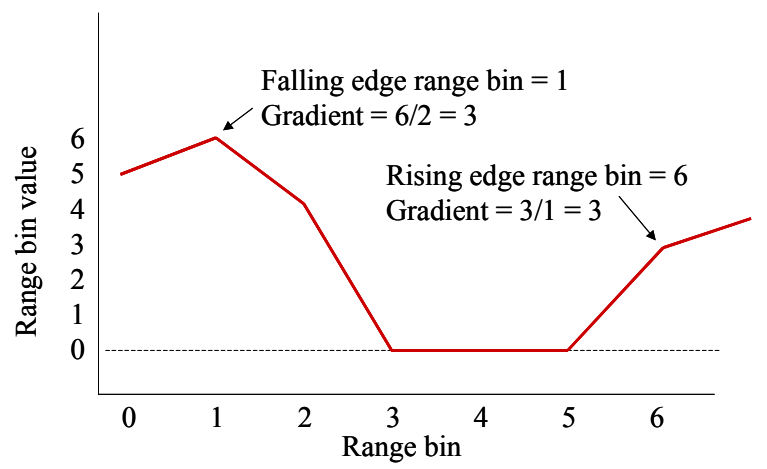

Figure 3. Segment of sample radial measurement and shadow edge features

At this point in the algorithm, one-to-one disparity checking could be performed since both threads have derived features in the same frame of reference. For example, comparison of rising edge or falling edge range bins along each radial could be used as the disparity metric. However, due to the nature of the measurements, it is expected that significant missed detections and false alarms will occur using this approach. In addition, by only looking at range bins where edges are detected, the approach does not take advantage of all the range bin values that do agree in the shadowed regions. For example, consider a large shadow region along a given radial. 
WxR-derived measurement

\begin{tabular}{|l|l|l|l|l|l|l|l|l|l|l|l|l|l|l|l|l|l|l|l|l|l|l|l|l|l|}
\hline 1 & 2 & 3 & 2 & 1 & 0 & 0 & 0 & 0 & 0 & 0 & 0 & 0 & 0 & 0 & 0 & 0 & 0 & 0 & 0 & 2 & 3 & 3 & 3 & 3 & 3 \\
\hline
\end{tabular}

DEM-derived measurement

\begin{tabular}{|l|l|l|l|l|l|l|l|l|l|l|l|l|l|l|l|l|l|l|l|l|l|l|l|l|l|}
\hline 1 & 1 & 1 & 1 & 2 & 2 & 2 & 0 & 0 & 0 & 0 & 0 & 0 & 0 & 0 & 0 & 0 & 0 & 1 & 2 & 2 & 2 & 2 & 2 & 2 & 2 \\
\hline
\end{tabular}

\begin{tabular}{|lcc|}
\hline & $\begin{array}{c}\text { Leading } \\
\text { edge }\end{array}$ & $\begin{array}{c}\text { Trailing } \\
\text { edge }\end{array}$ \\
WxR-derived edge range bin & 3 & 22 \\
DEM-derived edge range bin & 7 & 20 \\
Absolute disparity (edges) & 4 & 2 \\
Absolute disparity (shadow center) & \multicolumn{2}{c}{0} \\
\hline
\end{tabular}

The above observation has led to a disparity checking function that uses "range to center of shadow" as the prototype test statistic for disparity checking.

To provide additional confidence to the estimate of disparity, multiple measurements can be accumulated in each thread and shadow size can be monitored with respect to azimuth coverage (i.e. width). This capability completes the initial prototype for disparity checking. A shadow size is selected, a priori, and is specified by its coverage, (number of azimuth measurements) $\mathrm{x}$ (number of range bins). The disparity checker monitors for shadows of this size and compares the center locations of these shadows coming from both the WxR-derived thread and the DEM-derived thread. As will be shown, the trade-off using this approach becomes choosing a large shadow size that may result in few detections and infrequent disparity checks versus choosing a small shadow size that may result in missed detections and false alarms.

To test the approach presented in this section, flight-testing in the JNU region was performed to acquire a comprehensive set of sensor data in an area of severe terrain.

\section{FLIGHT TEST ENVIRONMENT}

A flight test was performed in the vicinity of JNU from September 10 through September 17, 2002. The flight-test aircraft was Ohio University’s King Air C90 (Figure 4).
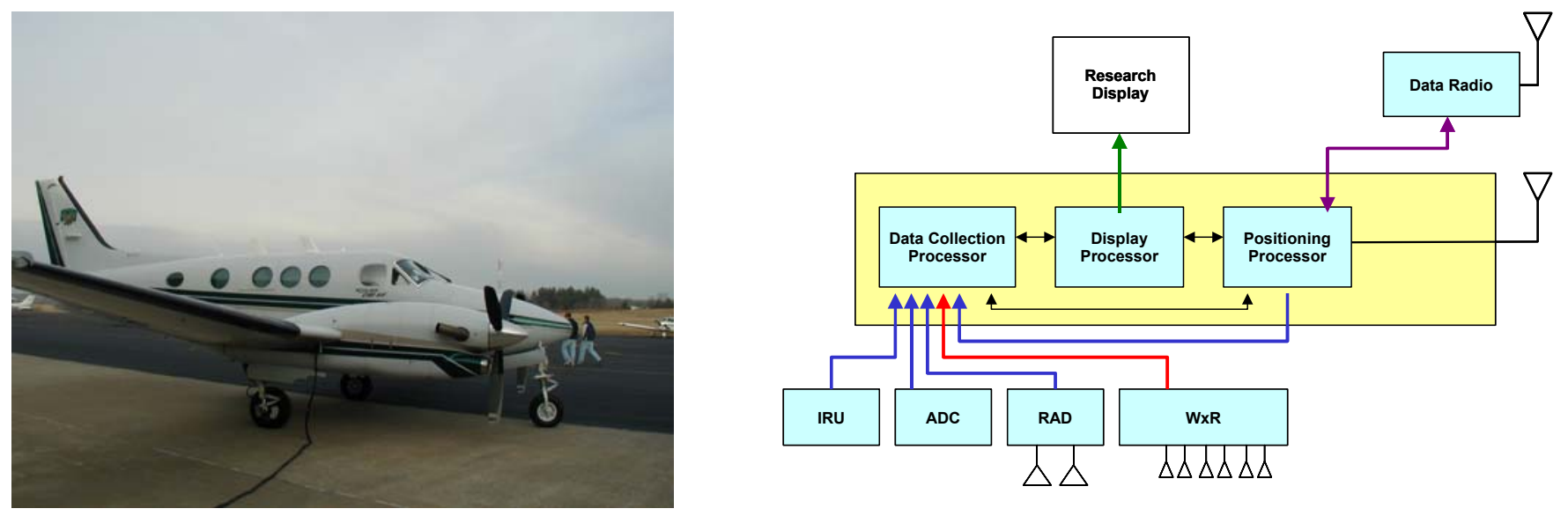

Figure 4. (a) Ohio University King Air C90, and (b) block diagram of flight-test equipment 
The experimental system used for this test is shown in Figure 4. Ranging measurements were provided by a commercially certified Bendix RS-811A WxR and a King KRA405B Radar Altimeter (RAD). Attitude and airspeed measurements were provided by an IRU and an Air Data Computer (ADC), respectively. Accurate positioning measurements were provided by a Positioning Processor consisting of a Novatel Euro-4 receiver and a Condor Engineering ARINC 429/453 interface card. The Positioning Processor was capable of using corrections provided by either the Wide-Area Augmentation System (WAAS) or a Local-Area Augmentation System (LAAS) prototype ground station via a data radio. The display processor receives aircraft state information from the other processors and combines it with a locally stored DEM to provide a virtual visual environment to the pilot on a flat panel liquid crystal display (LCD). This display software was developed by Delft University of Technology and is used through a memorandum of agreement. Figure 5 shows the installation of the display on the right side of the King Air's flight deck. The Bendix WxR display is shown just to the left of the experimental LCD in Figure 5.
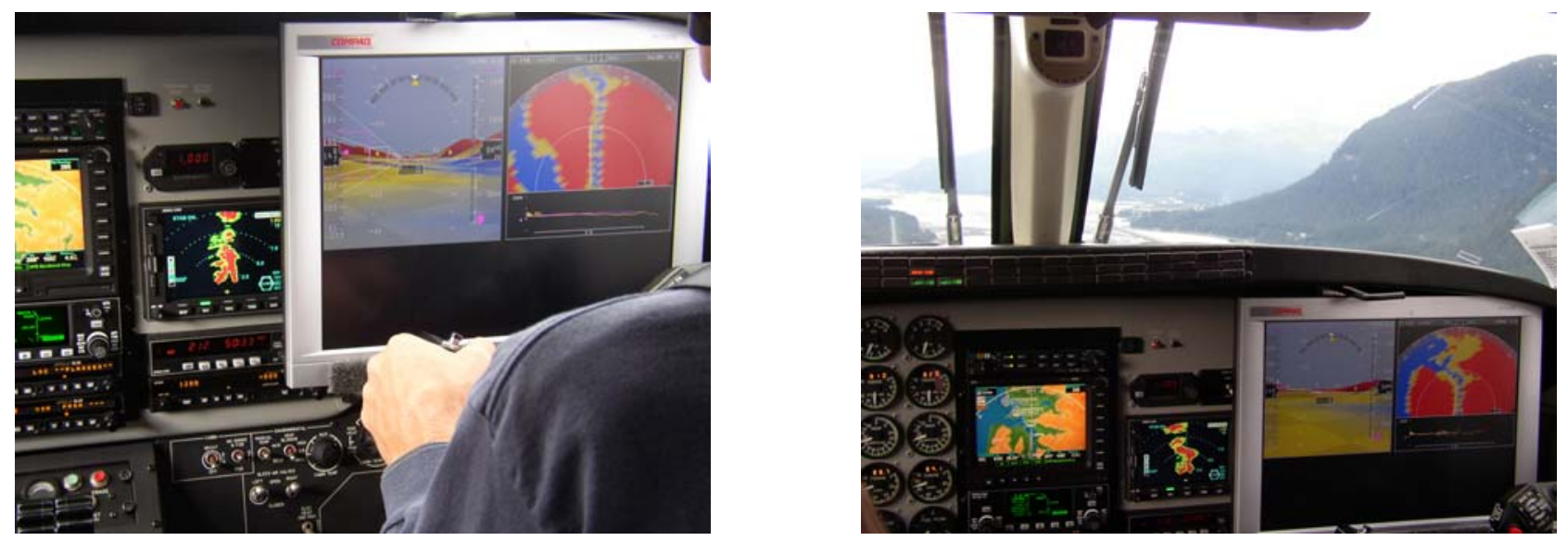

Figure 5. Installation of Synthetic Vision LCD for co-pilot in the King-Air flight deck.

The photographs shown in Figure 5 were taken while flying down the Gastineau channel approaching Runway 08 . At this point in the flight, the aircraft is below the terrain on either side of the flight path. This is indicated by the SVS display as red areas on the terrain. More importantly, note how these same areas are black on the WxR display. These shadow effects are what we seek to capitalize upon for the prototype integrity monitor algorithm.

A total of 41 approaches were flown to Runway 08 and Runway 26 at JNU (Figure 6).

\begin{tabular}{|l|c|c|}
\hline Date & Runway 08 & Runway 26 \\
\hline September 12 & 4 & 2 \\
\hline September 13 & 4 & 11 \\
\hline September 14 & 3 & 3 \\
\hline September 15 & 1 & 5 \\
\hline September 16 & 5 & 3 \\
\hline Total & 17 & 24 \\
\hline
\end{tabular}

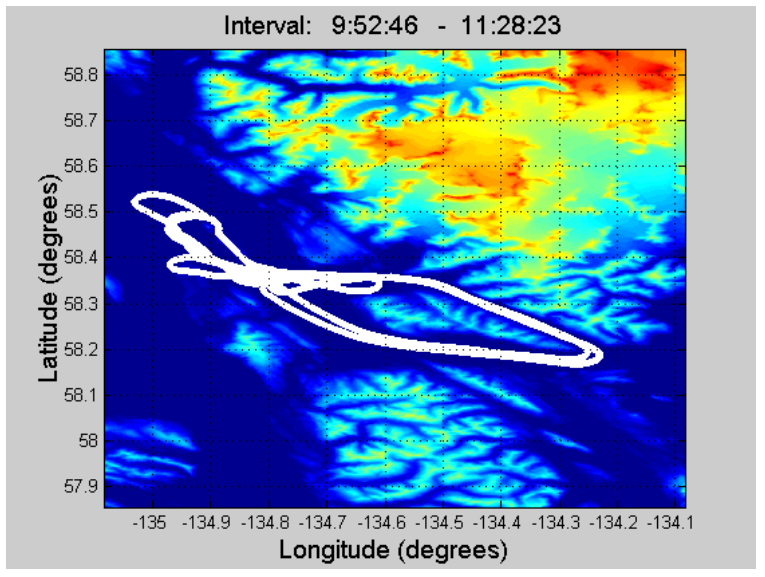

Figure 6. (a) Approaches flown during flight-testing, and (b) selected flight profiles 


\section{RESULTS}

Using the data collected at JNU, the prototype algorithms have been tested to validate the approach. To illustrate the processing steps described in Section 3, consider a representative scan taken on September 12 while flying down the Gastineau channel approach Runway 08. The WxR measurements are shown in Figure 7a. For all data collected at JNU, the scan range was set to $+/-45$ degrees and range was set to $10 \mathrm{nmi}$. The four color levels indicate increasing reflectivity levels detected by the WxR ranging from black (no detectable reflectivity) to blue to yellow to red (maximum reflectivity). Figure $7 \mathrm{~b}$ depicts relative terrain heights extracted from the DEM over the same coverage area. Red indicates terrain is higher than the aircraft altitude. The other contours are at decreasing levels to black. Black areas of terrain are greater than 250 meters below the aircraft altitude. From Figure $7 \mathrm{~b}$ the Gastineau channel is apparent in the DEM along with several tributaries that feed the channel.

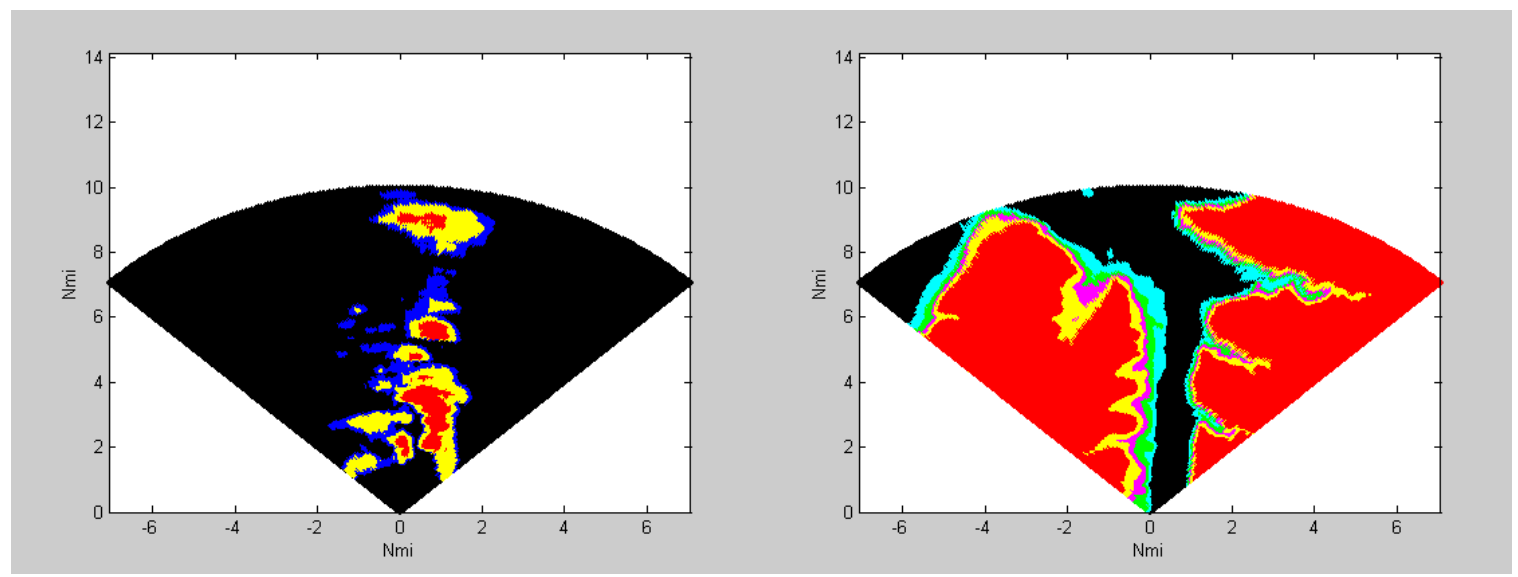

Figure 7. (a) WxR measurements, and (b) DEM contours within WxR field-of-view

Following the algorithm proposed in Section 3, the first step in each thread is to detect edge features along each radial and classify these edges. For the sample scan shown in Figure 7, the results of feature detection and classification are shown in Figure 8 for each thread. Visually there appears to be good agreement for this scan. However, as described in Section 3, comparing these edge locations directly will not be a good indicator of the actual parity (agreement), as it does not consider the agreement of all of the shadow cells between the edges that do agree.

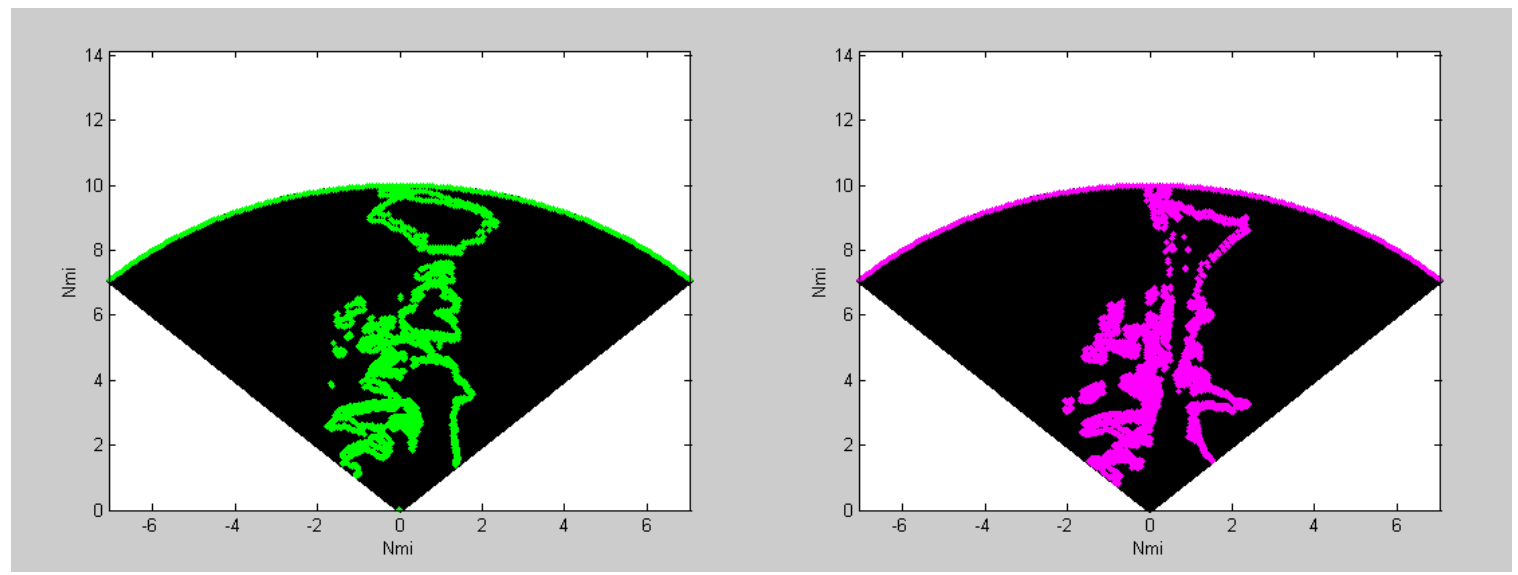

Figure 8. (a) WxR-derived edge features, and (b) DEM-derived edge features 
Figure 9 shows the locations of shadow center points for two sizes of shadows and the resulting disparity. These shadow center points were computed as described in Section 3. Sizes were chosen to be (13 x 55) and $(52 \times 110)$. These sizes correspond to ( $3 \mathrm{deg} \times 1 \mathrm{nmi})$ and $(12 \mathrm{deg} \times 2 \mathrm{nmi})$, respectively. Figures $9 \mathrm{a}$ and $9 \mathrm{~b}$ provide three distinct plots to illustrate the resulting effect on disparity checking. The top two plots represent the WxR-measurements (top) and the DEM-derived measurements (middle) in an image-space defined by the number of range bins ( 1 to 512) and the number of radial samples (1 to 384 ). 384 is the number of azimuth radial measurements that are made as the antenna scans from -45 degrees to +45 degrees and back again. In other words, the $\mathrm{x}$-axis is time and the y-axis is range. The pink pixels are those points in the images that have been found to be center points for the shadow sizes specified. When comparing Figure 9a to Figure 9b, significantly fewer shadow center points are detected when using a larger shadow size, as expected.

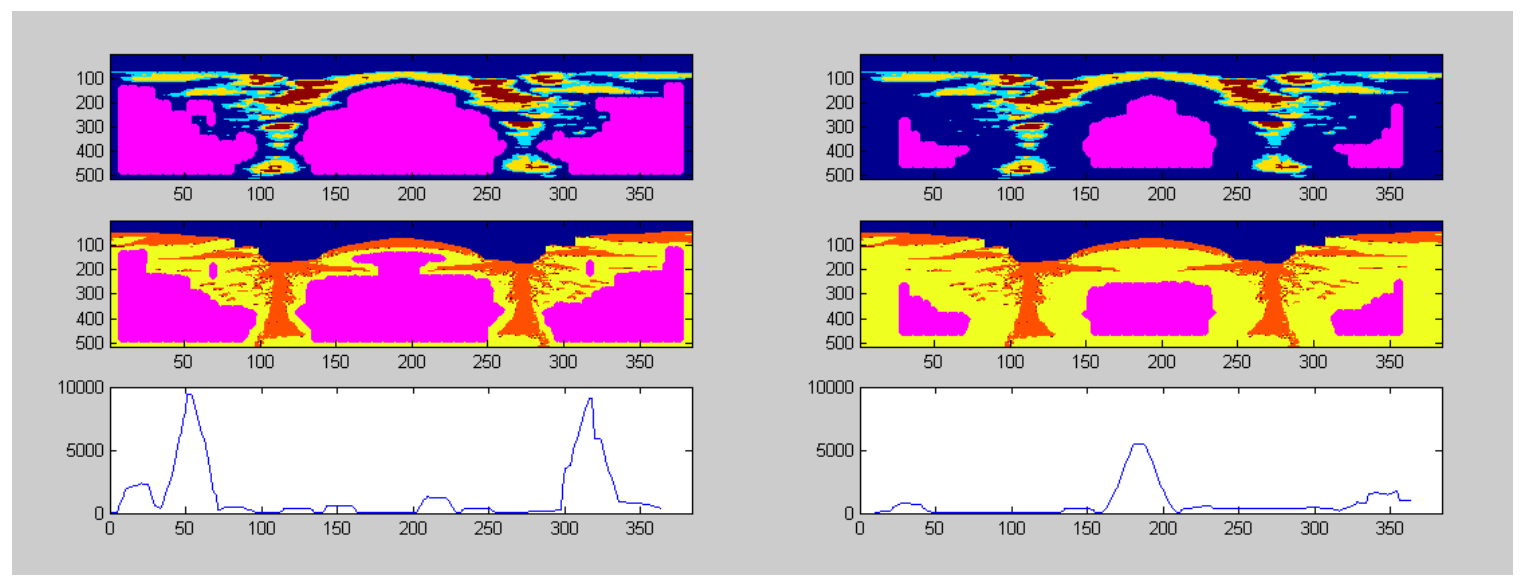

Figure 9. Measurements, shadow center points, and MSD disparity for shadow sizes

(a) 3 degrees by 1 nautical miles, and (b) 12 degrees by 2 nautical miles

The bottom plots in Figure 9 provide an example of a disparity metric that could be used by the integrity monitor. For this example, the Mean-Square Difference (MSD) is computed to the first shadow center point along each radial. MSD is computed using the following equation:

$$
M S D=\left(\frac{1}{N}\right) \sum_{i=1}^{N}\left(c_{W x R}[i]-c_{D E M}[i]\right)^{2}
$$

where $N$ is the number of samples and $c$ is the center point range bin for the first shadow found along radial $i$ by the WxRderived thread and the DEM-derived thread. The number of samples used for the MSD is 20 for the plots in Figure 9. Notice the effect on the MSD when using a larger shadow size.

Finally, detection of DEM errors is tested. For the sample scan shown previously, Figure 10 provides plots of the MSD disparity metric in the presence of biases inserted into the DEM. The number of samples used to compute the MSD for these cases is 50. For Figures 10a and 10b, the bias ranges from 0 (top), to $200 \mathrm{~m}$, to $400 \mathrm{~m}$, to $800 \mathrm{~m}$, and to $1600 \mathrm{~m}$ (bottom). For Figure 10c, the bias ranges from 0 (top), to $100 \mathrm{~m}$, and to $200 \mathrm{~m}$ (bottom).

Referring to Figure 10, using a detection threshold of 20000, the monitor will detect disparity in the presence of an 800-meter along-track bias in approximately 180 samples. At a measurement rate of $6 \mathrm{msec}$, this corresponds to about 1.08 seconds. The monitor will detect a 100-meter cross-track bias in approximately 200 samples (1.2 seconds), and a 200 -meter vertical bias in approximately 140 samples ( 0.84 seconds). In addition, it is apparent that as the bias becomes larger, it will be detected sooner.

Figure 10 also indicates the type of terrain we are flying over. In this particular scan while flying along the channel, edges are primarily vertical, or along-track. This makes disparity detection much more sensitive to cross-track errors, while alongtrack errors are more difficult to detect. 


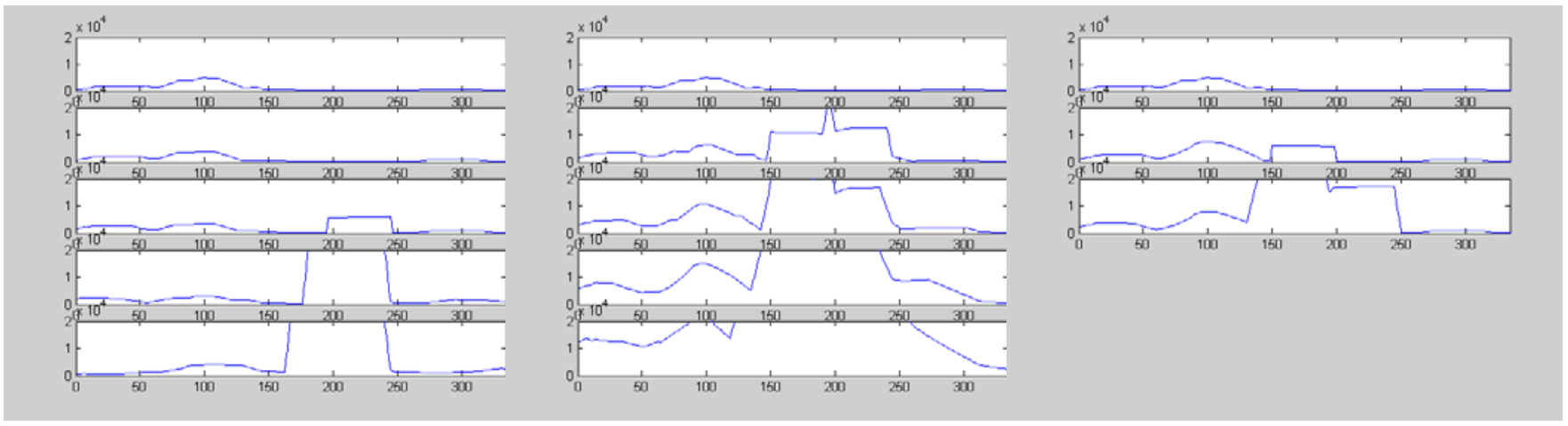

Figure 10. MSD disparity metric in the presence of DEM bias (a) along-track, (b) cross-track, and (c) vertical

\section{DISCUSSION}

Preliminary results indicate that the proposed algorithm has the potential to enable the use of commercial-of-the-shelf (COTS) X-band WxR systems for real-time DEM integrity monitoring; however, several issues remain that will be addressed as the research continues. These include (1) performing operational assessments, and (2) determining optimum detection and disparity metrics.

\subsection{Operational assessments}

Operational assessments are important in order to fully characterize the flight environment with respect to the features that may be used for disparity checking. The current algorithm assumes shadowing effects will be observable in WxR measurements. This will be a function of terrain, WxR antenna pointing direction, and proximity to the terrain. From the JNU data, flight segments at the higher altitudes do not provide results as good as the segments at the lower altitudes. This is primarily due to the loss of angular resolution at the longer ranges, but also due to the fact that at altitude, it is less likely that the WxR antenna beam pattern is illuminating terrain. Fortunately, phases of flight requiring high integrity for SVS DEMs will be when the aircraft is close to terrain (e.g. approach and departure phases). By triggering the integrity monitor operation at a prescribed altitude above the ground (e.g. 2000 feet), the concern becomes alleviated. The use of radar altimeter measurements to provide this triggering seems appropriate.

A second issue to address during operational assessment is real-time feasibility. A real-time implementation of the proposed algorithm is being developed and will be tested during the summer of 2003 in the Nevada area using NASA's B-757 test aircraft equipped with the Rockwell-Collins WxR-2100. The most significant real-time challenge will be accessing the DEM and generating the DEM-derived radial measurements every $6 \mathrm{msec}$ for comparison with the $\mathrm{WxR}$ radial measurements. One mitigation strategy is to perform less frequent comparison of features at the expense of longer detection times.

Lastly, quantifying nominal sensor errors in the operational environment is needed to establish minimum detectable DEM errors and to determine appropriate disparity thresholds for the integrity monitor.

\subsection{Optimal detection and disparity metrics}

Several options are being considered to optimize the feature detection method. Initially, it is proposed to choose a shadow size prior to flight and derive a disparity test statistic and alert thresholds based on this size. An alternative approach would be to use a dynamic method. Using this approach, shadow size may be used as the disparity metric.

With fixed shadow sizes, there is a distinct trade-off between small shadows and large shadows. As shadow size increases, there will be fewer, if any, center points to use for disparity checking; however, for the points that are found, the likelihood of agreement will increase. The disadvantages of using large shadows are (1) small errors will not be as detectable, and (2) as shadow size increases, the likelihood of the WxR observing one in a particular scan will decrease. Thereby, reducing the availability of the monitor. 
On the other hand, using small shadows will detect small disparities (e.g. a peak that is missing in the DEM), but will also provide more false alarms due to nominal noise-like errors in the WxR measurements and the DEM. One behavior that is common in the JNU data when using small shadow sizes is a detection by one thread and not by the other. Disparity checking in this case will result in a large difference that may lead to a false alert. To mitigate this behavior, repeated scans can be accumulated to see if the feature persists in one thread and not the other. If it does, this indicates an actual error that should be detected. Using multiple scans in this way will improve integrity but must be traded against the increase in timeto-alarm that results from waiting for additional scan measurements. The other benefit of using small shadows is that as shadow size gets smaller, the likelihood of observing shadows will increase. This increases the availability of the integrity monitor.

Finally, the choice of disparity metric must be made using a statistical approach. This allows the resulting integrity to be quantified and/or bounded as is often required for certification. The use of MSD has been shown to be effective for radar altimeter implementations of a DEM integrity monitor. Unfortunately, the forward-looking sensor approach suggested in this paper must consider nominal sensor errors that were not included in the radar altimeter statistical derivation of disparity test statistics and appropriate thresholds. These include attitude errors coming from the IRU, antenna pointing direction errors, and the ranging sensor signal-in-space characteristics. The nominal effect of all of these errors on the features extracted from both threads must be well understood in the fault-free case in order to set appropriate thresholds to indicate the detection of off-nominal, or faulty, cases.

\section{SUMMARY}

An approach to real-time DEM integrity monitoring using a forward-looking sensor has been presented. This approach requires a sensor that makes ranging measurements during scanning. Also required is a positioning system and an attitude and heading reference system. Using these systems, edge features are detected and extracted from the scanning ranging sensor. DEM-derived edge features are also detected and extracted in a parallel thread. Disparity checking is performed over areas between the detected edges. An example implementation using X-band Weather Radar is presented that uses shadow areas (i.e. regions between edges with zero reflectivity) for disparity checking. The prototype algorithm has been tested against data acquired in Juneau, Alaska, and the results suggest the approach is valid, but additional work is required to establish optimum performance and to verify real-time feasibility.

\section{ACKNOWLEDGEMENTS}

The authors would like to thank the Ohio University King-Air pilots, Brian Branham and Jamie Edwards, and the crew chief, Jay Clark, for their support and expertise during the flight-testing at JNU. The work presented in this paper was supported and partially funded through NASA under Cooperative Agreement NCC-1-351.

\section{REFERENCES}

1. "International Standards and Recommended Practices - Aerodromes", ICAO Annex 14, International Civil Aviation Organization, July 1999.

2. "Standards for Processing Aeronautical Data", DO-200A, RTCA, September 1998.

3. Uijt de Haag, M., Young, S., Sayre, J., Campbell, J., and Vadlamani, A., "DEM Integrity Monitor Experiment (DIME) Flight Test Results", International Society for Optical Engineering (SPIE) Aerosense Conference, Orlando, Florida, April 1-5, 2002.

4. Uijt De Haag, M., Young, S., And Gray, R., "A Performance Evaluation of Elevation Database Integrity Monitors for Synthetic Vision Systems", 8th International Conference on Integrated Navigation Systems, Saint Petersburg, Russia, May 28-30, 2001.

5. Gray, R. A., "In-flight Detection of Errors for Enhanced Aircraft Flight Safety and Vertical Accuracy Improvement Using Digital Terrain Elevation Data with an Inertial Navigation System, Global Positioning System and Radar Altimeter," Ph.D. Dissertation, Ohio University, Athens, Ohio, June 1999.

6. "Airborne Weather Radar with Forward Looking Windshear Detection Capability", ARINC Characteristic 708A-3, ARINC, November 15, 1999. 
7. Dieffenbach, O., APALS ${ }^{\mathrm{TM}}$ Autonomous Precision Approach and Landing System, Proceedings of the Society of PhotoOptical Instrumentation Engineers (SPIE), Orlando, FL, Apr. 18-19, 1995.

8. Ammar, D., "Radar Based Terrain and Obstacle Alerting System", U. S. Patent Number 5,945,926, U. S. Patent Office, August 31, 1999.

9. Morici, M., "Aircraft Position Validation using Radar and Digital Terrain Elevation Database", U. S. Patent Number 6,233,522, U. S. Patent Office, May 15, 2001.

10. "Minimum Operational Performance Standards for Airborne Weather and Ground Mapping Pulsed Radar", RTCA Document DO-173, RTCA, November 19, 1980.

11. "Minimum Operational Performance Standards for Airborne Weather Radar with Forward-Looking Windshear Detection Capability", RTCA Document DO-220, RTCA, September 21, 1993. 